M. $R \operatorname{eg} u l a$

\title{
ZUR ANALYSE DER FÜGUNG CE FRIPON DE VALET
}

Alle bisherigen Deutungen des Syntagmas ce fripon de valet sorgfältig iiberprüfend, gelangt $\mathrm{S}$ t. L ye r in einer historisch und psychologisch unter. bauten Sonderstudie: Les appositions romanes du type li fel d'anemis, ce fripon de valet (ZRPh, LVIII, S. 348-359) zu dem iberzeugenden Ergebnis, dass die erwähnten Fügungen nicht als Fortsetzung der lat. Wendungen scelus viri, flagitium hominis, monstrum mulieris, sondern als Neuschöpfung der Affektsyntax zu betrachten sind. Auch darin wird man dem Verfasser beipflichten müssen, dass die Fùgung ce fripon de valet nicht mit dem in mehrfacher Hinsicht abweichenden Typ la ville de Paris auf einen Plan gestellt werden kann. Wenn ich trotz der in den Hauptpunkten bestehenden ẗbereinstimmung dasselbe Problem nochmals aufrolle, geschieht es, um nach Beseitigung einer Unvollkommenheit in der Strukturanalyse eine eindeutige Diagnose der Satzgliendschaft beider Elemente zu gewinnen.

Mein Widerspruch regt sich vor allem gegen die Bezeichnung sapposition«. Wenn die der reflektierten, affektfreien Syntax angehörige Wendung ce valet fripon lautet, kann in der affektbetonten Inversion weder valet noch fripon "Apposition« sein. Fripon ist, wie der Verfasser richtig erkannt hat, Prädikat in emphatischer Stellung. Eine Apposition (= Anfügung) ist eine periphere Prädikatsverdichtung, die den Begriff ihres Beziehungswortes erläutert, aber nicht qualifiziert. Ausserdem ist sie eine horizontale "Mitfügung", wie sie Franz Houdek charakterisiert. In ce fripon de valet! liegt zentrale setzung vor. Es ist wie syntaktisch gleich gegliedertes und stilistisch gleichfarbiges Quel fripon que ce valet! ${ }^{1}$ ein affektbetonter Betrachtungsitz.

Was den Anzeigewert von de betrifft, so sieht der Verfasser darin "un simple signe de rapport appositionnelw, eine Deutung, die nach dem bisher Dargelegten einer Korrektur bedarf. De kennzeichnet die Un t e r or d$\mathrm{n}$ ung des unmittelbar folgenden Satelliten. Wenn Ly e $\mathrm{r}$ ce fripon de valet

${ }^{1}$ Que ce valet ist, sprachgeschichtlich gesehen, ein elliptischer Relativsatz. Nyrop lässt ihn aus que (est) ce valet entstehen. Vgl. Ce que c'est que la vie! Q ue, ursprïnglich prädikatives neutrales Relativ, ist zu einem sprachlichen Zeichen des syntaktischen Einschnittes zwischen Prädikat und Subjekt geworden. H. Frei sprcht in seiner Grammaire des fautes, p. 272 von einer "pause prononcéer. Unmöglich erscheint mir die Deutung dieses que als nconjonction« in den Werken von $\mathrm{M} \mathrm{i} \mathrm{chaut/Schricke,pp.} \mathrm{375,} \mathrm{537,} \mathrm{Lan} \mathrm{us} \mathrm{se/Yvon,} \mathrm{§} 287$ und De B oer, Synt, du Français moderne, pp. 127, 238). 
mit urbs Romae parallelisiert, gerät er zu seinen eigenen Aufstellungen in Widerspruch. Romae ist a p p ll at i ver Genetiv, während das nur formlich entsprechende de valet nach des Verfassers Ansicht, der man unbedenklich zustimmt, kein sgénitif appositionnel (S. 349), sondern S u b j e k t ist (S. 352) $D e$ stellt die logische Beziehung (nach L. Te sniè re die "c o nn ex $x$ i on") zwischen dem emphatisch gesetzten Prädikat (ce fripon) und dem psychologischen Subjekt her, das in diesem Fall zugleich das logische ist. Als Präposition des Betref $\mathrm{f}$ kennzeichnet de in anschaulich-expliziter Weise valet als jenes Satzglied, von dem das affektvoll herausgeschleuderte ce fripon ausgesagt erscheint. ${ }^{2}$ Weniger überzeugend ist die Erklärung von de als pausenfüllendem, rhythmischem Element zur modulatorischen und psychodynamischen Abhebung zweier syntaktisch verschiedener Satzglieder. C. $M$. R ober.t, Grammaire française, Paris, 1929, p. 124 ff. vergleicht dieses de nicht übel mit dem in Sätzen vom Typ: Il y a plusieurs soldats de blessés, doch besteht,' abgesehen von der verschiedenen Stilebene, ein Unterschied im Funktionswert: valet ist logisches $S \mathrm{ub} \mathrm{j} \mathrm{e} \mathrm{kt,} \mathrm{blessés} \mathrm{dagegen} P \mathrm{rädikat}$ i v.

Es fragt sich nun, ob de valet nicht auch als Genetiv des Eigenschaftsträgers (génitif au qualifié) gelten kann. Der Nominativ in li fel d'anemis, li prouz d'on (neben älterem prouz d'omne) liesse sich als constructio ad sensum erklären. De valet wäre demnach ein "Attributsub jektu.

Die Artikellosigkeit des Attributs erklärt sich nicht aus dem vermeintlich ursprünglichen icaractère exclamatif», wie der Verfasser meint; denn die von ihm angenommen "mononòmes" (Quel) fripon! valet! ( = Vokative) sind keine Vorformen, sondern rein theoretische Konstruktionen. Es liegt vielmehr verallgemeinernde Erfassung des Merkmalsträgers vor - lediglich Nennung der Begriffssphäre - wie in un bout d'homme, une énormité de maison, ebenso in Fällen des de qualificatif: une intelligence de café-concert, de samedi de paie (vgl. iRindfleischhorizontu), la tâche de frère aîné, le rôle d'oncle, l'air de juge u. a.

Quel fripon de valet beđeutet demnach: Quel fripon qu'un (tel) valet! "Was für ein Spitzbub - so ein Diener! $\iota^{3}$. In der ungebrochenen Form erscheint auch im Französischen der unbestimmte Artikel: une maîtresse chèvre, un maître âne, worin das Fehlen des de auffällt.

${ }^{2} D e$ als $\mathrm{Subjektszeichen} \mathrm{hat} \mathrm{A.} \mathrm{Tobler} \mathrm{für} \mathrm{das} \mathrm{Alfranzösische} \mathrm{er-}$ wiesen. Als Marke des Betreffssubjekts begegnet es bereits im Lat.: $d e$ c e teris senatui curae fore (Sallust, bell. Iug. 26, 1). Nfrz. :C'est joli, de la place ou nous sommes (Daudet, Rose et Ninette). GIos ierendes Funkti ons denken begegnet beim präpositionalen Akkusativobjekt im Spanischen und Rumänischen, bei de als Prädikativzeichen: Si j'étais (que) de vous;et d'un et de deux; - en voilà bien d'une autre;- et tous de rire (= voila tous riant);nous avons un voisin de malade, un bras de libre, une page d'ecrite.- Je crois que vous n'êtes pas 'de ces commis voyageurs de Paris qui lisent leur journal de couchés (J. Aicard, Les deux Stablazaïres) mit provenzalischem de der Körperstellung; Il est d'usage, $d e$ toute rigueur (vgl. lat. moris, consuetudinis est);- $d e$ plus, d'ailleurs. - Plastisches Kasusdenken liegt den lat. Umschreibungen mit $a b$, $a d$ und de zugrunde.

${ }^{3}$ Hieher gehört auch Pauvre de moi! vgl. sp.: Desdichado de mi! 
Bezuiglich der psychodynamischen Abhebung und der damit verbundenen rhythmischen Brechung wäre die Wendung En voilà une, d'idèe zu vergleichen. Es besteht hier kein partitives Verhältnis, sondern Retouchierung des in seiner Beziehung unbestimmten, absolut gebrauchten une. Für dieses Syntagma hat die von $R$. F. M i k u š vorgenommene Analyse der zweigliedrigen Fügungen Geltung. Nur möchte ich statt "Identifikations-« und "Unterscheidungsglied" die Termini "Grund-" und "Bestimmungsglied" gebrauchen.

Die Sonderheit der erörterten Nexusart $t^{4}$ besteht darin, dass der Merkmalsträger (valet), der in der aufgelösten Form der Fügung das grammatische und psychologische Subjekt bildet, in der Verdichung zum Attribut herabsinkt,' während das ausgesagte Merkmal (fripon) zum Kern- oder Leitglied beförđert wird: ein syntaktisches Paradox!

Graz, Mai 1965.

\section{P O V Z E TE}

Prispevek $\mathrm{k}$ analizi konstrukcije Ce fripon de valet

Afektivno sintagmo Ce fripon de valet sestavljata dejanski predikat, ki je postavljen na začetek, in pripadajoči mu (logični) subjekt, kateremu jemlje podredni . de prvotno vrednost, $\mathbf{s}$ tem da ga formalno napravi $\mathrm{za}$ atribut - kar je pravi sintaktični paradoks. Podobno fungira $\mathrm{v}$ formuli $E n$ voilà une d'idée sicer proklitični člen (une) kot uregensu, medtem ko ima z njim zvezani substantiv vlogo podrejenega dela sintagme.

4 Die von 0 . Jespersen auf die Subjekt-Prädikatbeziehung eingeschränkte Bezeichnung iN ex.u si kann auf jedes Syntagma angewendet werden. 